\title{
Transformation of passive electrical networks with distributed PWL-sources using a Model Order Reduction
}

\author{
S. Ludwig ${ }^{1,2}$ and W. Mathis ${ }^{1}$ \\ ${ }^{1}$ Leibniz University Hannover, Institute of Electromagnetic Theory, Hannover, Germany \\ ${ }^{2}$ Fraunhofer Research Institution for Electronic Nano Systems, Advanced System Engineering, Paderborn, Germany
}

\begin{abstract}
In modeling of distributed systems with distributed sources large networks with RLC-elements and independent sources arise. This high complexity leads to a high effort in simulations. Therefore model reduction can be used to reduce these networks, preserving the behavior at the observed nodes in the networks. For the reduction of networks with a large number of independent sources only a weak reduction is enabled with standard model reduction techniques. In this paper an efficient reduction of networks with a large number of sources with piece-wise-linear waveforms is presented, using the decomposition of piece-wiselinear functions. With the proposed method a higher reduction of the network and/or a higher accuracy can be achieved with model reduction. The validity and efficiency of the proposed method is shown by reducing a RCI-Grid model.
\end{abstract}

\section{Introduction}

Since the early beginnings of circuit theory similarity and equivalence transformation of electrical networks are of strong interest. In the modeling of distributed systems, such as electromagnetic or thermal fields, with distributed sources very large networks are created. For example in electromagnetic fields in ICs the switching currents act like distributed sources or in thermal simulations of substrates the transistors act like distributed heat sources. For these very large networks a smaller network with approximated port behavior is required to allow for fast simulations. With the help of model order reduction and network synthesis it is possible to find such a reduced network (Antoulas, 2005; Ionutiu et al., 2007; Ludwig et al., 2008a; Radic et al., 2008). Replac-

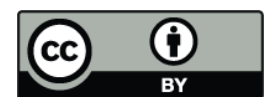

Correspondence to: S. Ludwig (ludwig@ tet.uni-hannover.de) ing the original network with the reduced network enables investigations with a lower effort for simulations.

In our former work we have developed an efficient reduction method for networks with groups of distributed sources which have equal or proportional amplitudes (Ludwig et al., 2008a,b,c). In this paper we extend this approach to sources with piece-wise-linear (PWL) waveforms (PWL-sources), which do not necessarily have equal or proportional amplitudes.

In this work the design flow for the approximative transformation of large passive electrical networks, which especially contain a high number of PWL-sources, is presented in Sect. 2. Firstly, the network is transformed with an equivalence-transformation in Sect. 2.1 to reduce the number of independent sources and to enhance the reduction process. Secondly, the network is reduced with an approximative transformation using model order reduction algorithms in Sect. 2.2. The efficiency of the complete reduction process is shown by reducing an example network in Sect. 3 and the paper is concluded in Sect. 4.

\section{Transformation of networks with distributed sources}

For the transformation of electrical networks the invariant as well as the approximative properties of the network have to be defined. Invariant properties are for example stability and passivity. Properties that can be approximated are the behavior at the nodes for the connection with other networks and electrical values that should be observed or measured. Also the behavior at the nodes where independent sources are connected can be approximated. Other properties, such as the number of elements or nodes or the behavior at nodes which are not of interest can be disregarded.

Distributed systems with distributed sources are modeled with passive RLC-elements a high number of independent sources. In state-of-the-art model reduction these 


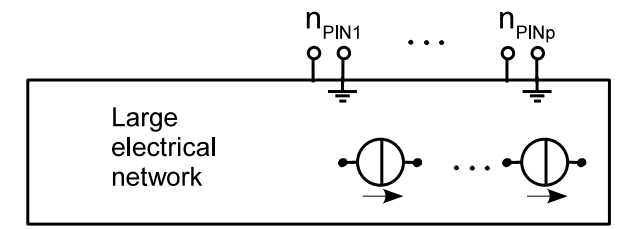

Extraction of distributed sources
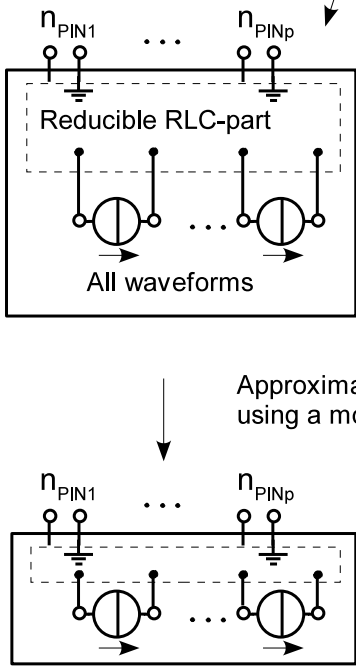

Small electrical network

Equivalence transformation

ve transformation del order reduction
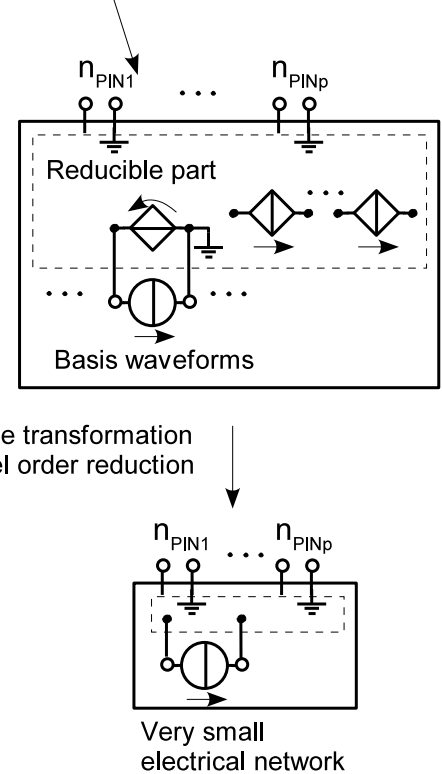

Fig. 1. Transformation flow for networks with distributed sources.

independent sources are extracted from the RLC-part of the network and connected through ports and only the RLC-part is reduced (left path in Fig. 1). The advantage of this extraction is that only passive RLC-elements are used in the reducible model and the passivity of the whole model can be preserved during a model order reduction (Odabasioglu et al., 1998). This leads to a passive reduced model and prevents unstable time-domain simulations. For networks with a large number of independent sources, this extraction produces a large number of ports. A large number of ports is a strong limitation for the model reduction (Silva et al., 2007b; Feldmann, 2004). In many distributed systems the waveforms of the sources are not completely independent of each other. For equal or proportional waveforms of the sources, which are widely used in modeling, we have developed a method to describe these sources efficiently for model reduction (Ludwig et al., 2008a,b,c). The idea is to reduce the number of independent sources in order to enable stronger reduction of the reducible part (dotted lines in Fig. 1) of the network. For these networks we propose an equivalence transformation (right path in Fig. 1) instead of the extraction of the sources. The basis-waveforms are given by one representative of each group and are realized with additional independent sources. The waveforms are copied into the network by replacing the distributed sources with controlled sources with a specific gain. As only the independent sources, representing a group of sources, have to be extracted and connected through ports, a network with a reduced number of ports is generated. The resulting network can be more efficiently reduced than the network with extracted sources (Ludwig et al., 2008a). The network generated in this way is generally not passive. The connection of non-passive systems can lead to unstable time-domain simulations and therefore a passive system is preferable. Controlled sources connected with the additional independent sources are added. These sources act like dissipative elements that dissipate exactly the amount of energy generated by the controlled sources in the network and vice a versa. Thereby the equivalent network is passive (Ludwig et al., 2008a,c) and passivity can be preserved in the reduced model with the description proposed in Ludwig et al. (2008c).

In models that are generated from measurements of distributed systems the sources in the model have PWLwaveforms, which are not necessarily equal or proportional. In the next section the described approach for an equivalence-transformation of the network is extended to networks with PWL-sources.

\subsection{Equivalence transformation for the reduction of the number of PWL-sources}

In this section we propose a method to describe PWL-sources in a network for an efficient reduction. We consider networks with sources, where the waveforms of the sources are described with continuous PWL-functions $f_{q}(t)$ on a closed interval $[0, T]$ for which holds

$f_{q}(t)=m_{i} t+o_{i}$ for all $a_{i-1} \leq t \leq a_{i}$

for pairs of real numbers $\left(m_{i}, o_{i}\right), i=1, \ldots, k$ with

$0<a_{0}<a_{1}<\ldots<a_{k}<T$

according to the definition in Aliprantis et al. (2005).

Every PWL-function describing a waveform of a source in the network is decomposed into PWL-basis-functions. The union of all basis-functions gives the complete basisfunctions space of the size $b$. The waveforms of all sources are now the linear combination of all $b$ basis-functions multiplied with a corresponding weighting factor $w_{i}$.

$f_{q}(t)=\sum_{i=1}^{b} w_{q, i} f_{i}(t)$

The basis-functions can be found for example with the method proposed in Aliprantis et al. (2005).

After their decomposition the PWL-functions have to be realized in the network. In the network every basis-function is realized with an independent PWL-source. The distributed sources in the network are replaced with controlled sources, with the controlling values and gains corresponding to the 
linear combination of the basis-functions as in Eq. (3). With this method a network with equivalent behavior at the pins of the network is generated, hence this modification is an equivalence-transformation as shown in the right path in Fig. 1.

If the number of basis-functions is lower than the number of distributed PWL-sources in the original network, with this method a network with a lower number of independent PWLsources can be generated. By extracting the lower number of independent PWL-sources a reducible network with a lower number of ports can be generated, which can be more efficiently reduced. Additionally, the reducible part of the network is still passive and reciprocal by adding appropriate controlled sources to the additional independent sources for the basis-functions, as described earlier in this section.

The difference compared to the former method for groups of waveforms with equal or proportional amplitude is that not for every group of waveforms an independent source is added but every basis-function is realized with an independent source. The distributed sources inside the network are again replaced by controlled sources, which are now not only controlled by the representative source for each group but by all additional independent sources.

\subsection{Approximative transformation using a Model Or- der Reduction (MOR)}

Model order reduction algorithms based on projection are commonly used for the reduction of large systems of electrical networks (Ionutiu et al., 2007). The advantage of the projection methods is the simple implementation and the low computational effort for the construction of the reduced model.

For MOR the electrical network of the model has to be described as a system of differential algebraic equations in the form of

$$
(s \mathbf{C}+\mathbf{G}) \mathbf{x}=\mathbf{B u}, \quad \mathbf{y}=\mathbf{L}^{T} \mathbf{x}
$$

using modified nodal analysis, where the number of equations $N$ is defined as the order of the system. $p$ is the number of ports. The positive definite system matrices $\mathbf{C}, \mathbf{G} \in \mathbb{R}^{N \times N}$ contain the stamps for capacitors, inductors and resistors. The input and output vectors $\mathbf{u}, \mathbf{y}$ are related with the system vector $\mathbf{x} \in \mathbb{R}^{N}$ through ports with the help of the matrices $\mathbf{B}, \mathbf{L} \in \mathbb{R}^{N \times p}, \mathbf{B}=\mathbf{L}$. The matrix transfer function $\mathbf{H} \in \mathbb{C}^{p \times p}$ of the system is given by

$\mathbf{H}(s)=\mathbf{L}^{\mathbf{T}}(s \mathbf{C}+\mathbf{G})^{-1} \mathbf{B}$.

Circuit equations of typical networks have system matrices that are very sparse, even though the order $N$ is quite large. For the reduction we use the approach proposed in Phillips and Silveira (2005). Firstly a number of real frequency points $s_{i}$ is defined and for every frequency point

$\mathbf{z}_{k}=\left(s_{k} \mathbf{C}+\mathbf{G}\right)^{-1} \mathbf{B}$

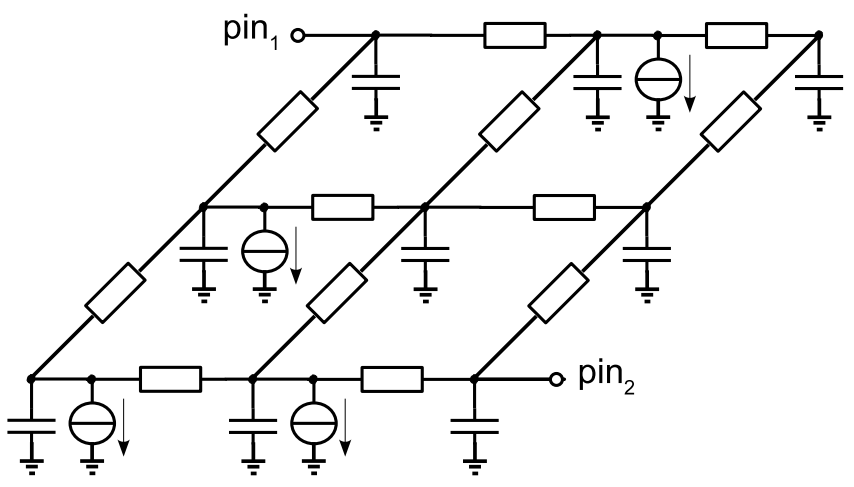

Fig. 2. Example of an $3 \times 3$ RCI-Grid model with 4 PWL-sources.

is calculated. By combining all $\mathbf{z}_{i}$ in $\mathbf{Z}=\left[\mathbf{z}_{1}, \mathbf{z}_{2}, \ldots, \mathbf{z}_{i}\right]$ and using a singular value decomposition

$[\mathbf{U}, \mathbf{S}, \mathbf{V}]=\operatorname{SVD}(\mathbf{Z})$

the projection matrix $\mathbf{T}$ is the orthogonal column span of $\mathbf{U}(:, 1: q)$ and the singular values in $\mathbf{S}$ can be used for choosing the reduced order and for error estimation (Phillips and Silveira, 2005), as only the dominant singular vectors corresponding to the $q$ largest singular values are used for the projection matrix. With the projection matrix $\mathbf{T}$ the system vector is changed with $\widetilde{\mathbf{x}}=\mathbf{T} \mathbf{x}$. The reduced system matrices are calculated with

$\widetilde{\mathbf{C}}=\mathbf{T}^{T} \mathbf{C T} \quad \widetilde{\mathbf{G}}=\mathbf{T}^{T} \mathbf{G T}$

$\widetilde{\mathbf{B}}=\mathbf{T}^{T} \mathbf{B} \quad \widetilde{\mathbf{L}}=\mathbf{T}^{T} \mathbf{L}$.

If the projection matrix is real, this method preserves the passivity of suitably described systems (Odabasioglu et al., 1998). The order reduced transfer function

$\widetilde{\mathbf{H}}(s)=\widetilde{\mathbf{L}}^{\mathbf{T}}(s \widetilde{\mathbf{C}}+\widetilde{\mathbf{G}})^{-1} \widetilde{\mathbf{B}}$

with $\widetilde{\mathbf{H}} \in \mathbb{C}^{p \times p}$ is generated, with an order $n \ll N$ of the system matrices. The reduced system closely matches the inputoutput-behavior of the original model, $\widetilde{\mathbf{H}} \approx \mathbf{H}$ (Phillips and Silveira, 2005).

In the last step for the approximative transformation of the network, the reduced system is synthesized as an electrical network, for example with a method described in Palenius and Roos (2004); Yang et al. (2007); Ludwig et al. (2008a).

With this transformation a smaller network can be found. In the resulting network, properties like passivity are preserved while the behavior at the pins is approximately the same as in the original network.

\section{Results for an example RCI-grid}

As an example a network grid as shown in Fig. 2 is used. The example grid contains resistors, capacitors and independent current PWL-sources. The 4900 resistors of our example are connected as a two-dimensional grid with $50 \times 50$ 

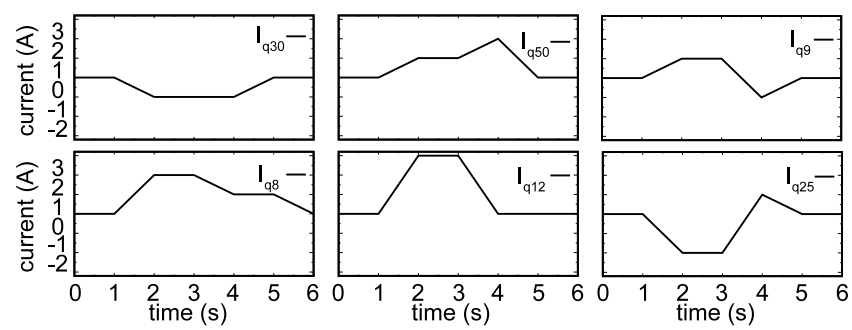

Fig. 3. Some waveforms of the current sources of the $50 \times 50$ RCIGrid model.

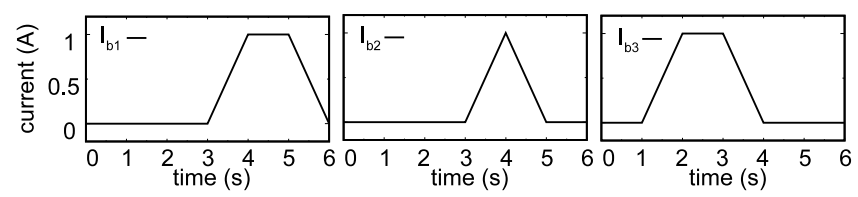

Fig. 4. Basis-waveforms for the $50 \times 50$ RCI-Grid model using decomposition of the original PWL-waveforms.

nodes and pins at two opposite corners of the network. Between every node in the $50 \times 50$ grid and the ground node a capacitor is connected, which amounts to 2500 capacitors. The values of the elements are randomly chosen in the ranges $1 \pm 0.5 \mathrm{ohm}$ and $0.1 \pm 0.05 \mathrm{~F}$ for the resistors and capacitors, respectively. Between 55 arbitrary chosen nodes in the network and the ground node independent current sources with PWL-waveforms are connected. Some examples for the PWL-waveforms used in the example are shown in Fig. 3. All waveforms can be decomposed into the three basis-waveforms shown in Fig. 4.

With the standard method of extracting all independent sources the system has 57 ports. If the waveforms of the independent sources are decomposed into the three basiswaveforms and the equivalence-transformation in Sect. 2.1 is used, only three independent sources are necessary. This results in only five ports of the reducible part of the network. With this description the behavior at the two pins of the model is not changed. Notably the system of the equivalence-transformed network can be, already before the reduction with MOR, calculated almost four times faster due to the lower number of ports (Table 1). Both system descriptions are reduced with the PMTBR algorithm as described in Sect. 2.2. One hundred expansion points $s_{i}$ are equally distributed in the frequency range range of interest $10^{-5} \leq f \leq 10^{5}$ and used for the generation of the projection matrix. In Fig. 5 the singular values $\mathbf{S}$ of the system with extracted sources and the equivalence-transformed system are shown. It can be seen that the singular values of the system after equivalencetransformation decay faster, which means that the reduced systems will be smaller and more accurate. For comparison both systems are first reduced to the same order of $n=40$. Secondly both systems are reduced to a maximal singular

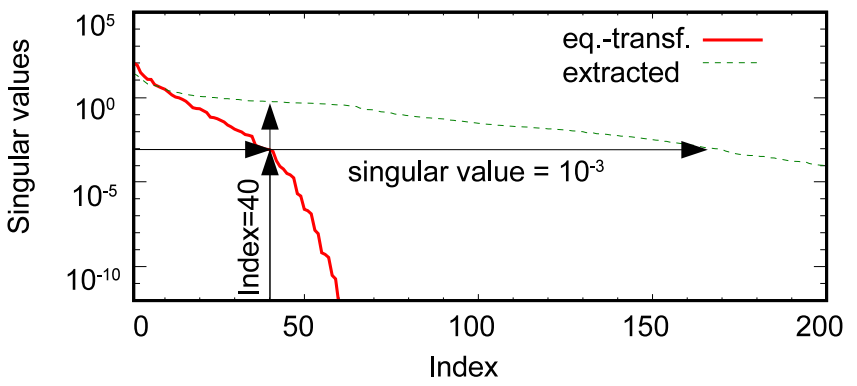

Fig. 5. Hankel singular values of the $50 \times 50$ RCI-grid.

Table 1. Comparison of reduction results of the system.

\begin{tabular}{ccccc}
\hline $\begin{array}{c}\text { Original order } \\
N=2500\end{array}$ & $\begin{array}{c}\text { Ports } \\
\text { Extracted }\end{array}$ & $\begin{array}{c}\text { Reduced } \\
\text { order } n \\
\text { (Reduction) }\end{array}$ & $\begin{array}{c}\text { MATLAB } \\
\text { Speed-up }\end{array}$ & Accuracy \\
\hline $\begin{array}{c}\text { sources } \\
\text { Extracted reduced } \\
\text { with PMTBR }\end{array}$ & 57 & 2500 & $1 \times$ & $\begin{array}{c}\text { Exact at } \\
\text { the pins }\end{array}$ \\
$\begin{array}{c}\text { Extracted reduced } \\
\text { with PMTBR }\end{array}$ & 57 & $\begin{array}{c}165 \\
(93.4 \%)\end{array}$ & $9.4 \times$ & $\begin{array}{c}\text { Very } \\
\text { Good }\end{array}$ \\
$\begin{array}{c}\text { Equivalence- } \\
\text { transformed }\end{array}$ & 5 & $\begin{array}{c}40 \\
(98.4 \%)\end{array}$ & $42 \times$ & Bad \\
$\begin{array}{c}\text { Equivalent reduced } \\
\text { with PMTBR }\end{array}$ & 5 & - & $3.8 \times$ & $\begin{array}{c}\text { Exact at } \\
\text { the pins }\end{array}$ \\
\hline
\end{tabular}

value of $10^{-1}$, resulting in an order of $n=40$ for the system of the equivalence-transformed network and $n=165$ for the system of the network with extracted sources. The possible MATLAB-speed-up of the reduction of both systems is shown in Table 1. The transfer functions of the reduced systems with the maximal singular value of $10^{-1}$ show a good agreement with the original system, as shown for the transfer function $Z_{11}$ in Fig. 6. However, since the reduced system of the equivalence-transformed network is smaller, it can be faster simulated than the reduced system of the network with extracted sources $(52 \times$ in comparison to $9.4 \times$, Table 1$)$. For the same order, the speed-up of the system with extracted sources is almost as high as for the equivalence-transformed system $(42 \times$ and $52 \times$, Table 1$)$. Nevertheless, the reduced system of the equivalence-transformed network is more accurate (Figs. 6 and 7). With this example it is shown, that the proposed method of equivalence-transformation with the decomposition of the waveforms as an additional step before the reduction leads to smaller and therefore faster models than the standard method of extracting all PWL-sources. Also it was shown, that for the same size of the reduced system our equivalence-transformation before reduction leads to more accurate models.

For investigations with SPICE-like simulators the smallest reduced system that is close enough to the original, which is 


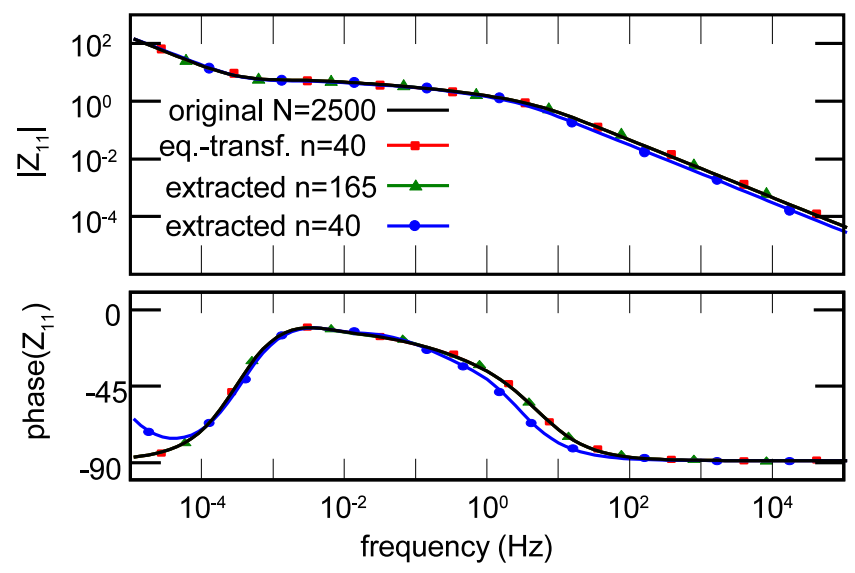

Fig. 6. Magnitude and phase of transfer function $Z_{11}$ of the $50 \times 50$ RCI-grid (MATLAB).

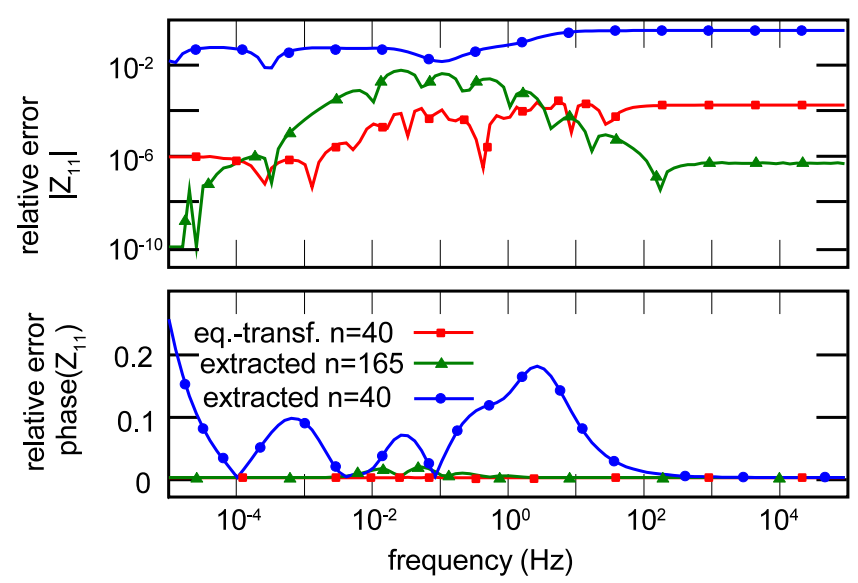

Fig. 7. Magnitude- and phase-error of transfer function $Z_{11} 50 \times 50$ of the RCI-grid (MATLAB).

the equivalence-transformed network reduced with PMTBR (last line in Table 1) is synthesized with the method described in (Ludwig et al., 2008a). The resulting network is smaller (Table 2) than the original network and simulations are around ten times faster. As an example the good agreement of the network behavior at the pins can be seen for a simulation of the voltage fluctuation at pin1 in Fig. 8.

\section{Conclusions}

In this paper, an efficient reduction process of networks with a large number of PWL-sources is presented. The former developed method of an equivalence-transformation instead of extracting sources is extended to PWL-waveforms which are decomposed into basis-functions. The validity and efficiency of the proposed method is shown by reducing a RCI-Grid model. With the proposed reduction process a higher reduction and a higher accuracy can be achieved. This results in
Table 2. Comparison of reduction results of the network.

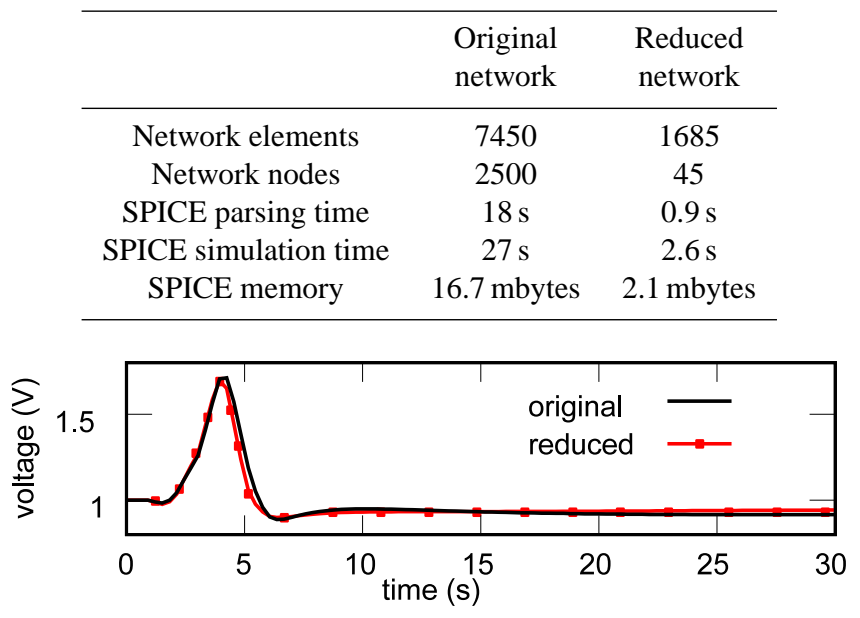

Fig. 8. Voltage drop at pin 1 of the $50 \times 50$ RCI-grid (HSPICE).

smaller and/or more accurate networks generated with the reduction process.

Acknowledgements. S. Ludwig wants to acknowledge Lj. RadicWeissenfeld and R. Kazemzadeh of the Institute of Electromagnetic Theory at the Leibniz University of Hannover for many useful discussions. Represented research and development work is carried out in the frame of the MEDEA+ project A701 PARACHUTE project This particular research was supported by the BMBF under $01 \mathrm{M}$ 3169 A, 01M $3169 \mathrm{D}$ and $01 \mathrm{M} 3169 \mathrm{E}$. The responsibility for this publication is held by the authors only.

\section{References}

Aliprantis, C. D., Harris, D., and Tourkey, R.: Continous Piecewise Linear Functions, Macroeconomic Dynamics, 10(1), 7799, 2005 .

Antoulas, A. C.: Approximation of Large-Scale Dynamical Systems, Adv. Design Control, SIAM, 2005.

Feldmann, P.: Model order reduction techniques for linear systems with large number of terminals, DATE'2004 - Design, Automation and Test in Europe, Exhibition Conference, vol. 2, Paris, France, 2004.

Ionutiu, R., Lefteriu, S., and Antoulas, A. C.: Comparison of Model Reduction Methods with Applications to Circuit Simulation, Scientific Computing in Electrical Engineering SCEE 2006, Mathematics in Industry, Springer, vol. 11, 2007.

Ludwig, S., Radić-Weissenfeld, Lj., Mathis, W., and John, W.: Efficient Model Reduction of Passive Electrical Networks with a Large Number of Independent Sources, IEEE International Symposium on Circuits and Systems ISCAS, Seattle, USA, 2008a.

Ludwig, S., Radić-Weissenfeld, Lj., Mathis, W., and John, W.: Efficient passive network description of IC conducted emission models for model reduction, Adv. Radio Sci., 6, 133-137, 2008b, http://www.adv-radio-sci.net/6/133/2008/.

Ludwig, S., Radić-Weissenfeld, Lj., Mathis, W., and John, W.: Passive and Reciprocal Network Description of Independent 
Sources for Efficient Model Reduction, International Conference on Signals and Electronic Systems - ICSES, Krakow, Poland, 2008c.

Odabasioglu, A., Celik, M., and Pileggi, L. T.: PRIMA: Passive Reduced- Order Interconnect Macromodeling Algorithm, IEEE Transactions on Computer-Aided Design of Integrated Circuits and Systems, 17(8), 645-654, 1998.

Palenius, T. and Roos, J.: Comparison of reduced-order interconnect macromodels for time-domain simulation, IEEE Transactions on Microwave Theory and Techniques, 52, 2240-2250, 2004.

Phillips, J. R. and Silveira, L. M.: Poor Mans TBR: A Simple Model Reduction Scheme, IEEE Transactions on Computer-Aided Design of Integrated Circuits and Systems, 24(1), 43-55, 2005.
Radić-Weissenfeld, Lj., Ludwig, S., Mathis, W., and John, W.: Model order reduction of linear time invariant systems, Adv. Radio Sci., 6, 129-132, 2008a, http://www.adv-radio-sci.net/6/129/2008/.

Silva, J. M. S., Villena, J. F., Flores, P., and Silveira, L. M.: Outstanding Issues in Model Order Reduction, Scientific Computing in Electrical Engineering, Springer, Berlin Heidelberg, Germany, 2007.

Yang, F., Zeng, X., Su, Y., and Zhou, D.: RLCSYN: RLC Equivalent Circuit Synthesis for Structure-Preserved Reduced-order Model of Interconnect, IEEE International Symposium on Circuits and Systems ISCAS, New Orleans, USA, 2007. 